\title{
Förderung der deutschen Sprache weltweit
}

Vorschläge, Ansätze und Konzepte

Herausgegeben von

Ulrich Ammon und Gabriele Schmidt

Unter Mitarbeit von

Birte Kellermeier-Rehbein

\section{DE GRUYTER}


ISBN 978-3-11-047670-5

e-ISBN (PDF) 978-3-11-047923-2

e-ISBN (EPUB) 978-3-11-047692-7

Library of Congress Control Number: 2019937569

Bibliografische Information der Deutschen Nationalbibliothek

Die Deutsche Nationalbibliothek verzeichnet diese Publikation in der Deutschen Nationalbibliografie; detaillierte bibliografische Daten sind im Internet über http://dnb.dnb.de abrufbar.

(C) 2019 Walter de Gruyter GmbH, Berlin/Boston

Druck und Bindung: $\mathrm{CPI}$ books $\mathrm{GmbH}$, Leck

www.degruyter.com 


\section{Inhalt}

Vorwort $-\mathbf{V}$

\section{I: Allgemeine thematische Beiträge}

Ulrich Ammon

Fördermöglichkeiten von Deutsch und Germanistik in der Welt im Überblick - 3

Gerhard Stickel

Zur Förderung von DaF aus der Sicht nationaler Sprachinstitute, speziell des Instituts für Deutsche Sprache (IDS) -25

Rudolf de Cillia

Die Förderung der deutschen Sprache und Österreichs Auslandssprachen- und -kulturpolitik - 37

Göz Kaufmann

Überlegungen zum Erhalt von (deutschen) Minderheitensprachen — 53

Christian Efing, Rudolf Hoberg

Sprachbildung und Sprachbewusstheit als Voraussetzung der

Sprachförderung: Die Dominanz des Englischen und ihre Folgen für das

Deutsche -71

Marianne Hepp

Deutschförderung durch Fachverbände — 87

Reiner Pogarell

Der Beitrag deutscher Unternehmen zur Verbreitung der deutschen

Sprache -107

Jan Kruse

Deutschförderung durch staatliche Medien - am Beispiel der Deutschen

Welle - 117 


\section{Deutschförderung durch Fachverbände}

\section{Einleitende Bemerkungen}

Verbände vertreten die Interessen ihrer Mitglieder und formulieren dafür verbindliche und längerfristige Ziele und Richtlinien. Verbände können Zusammenschlüsse von Einzelpersonen sein, oder können, wie im Fall der Dachverbände, mehrere, auch zahlreiche Verbände unter einem Dach zusammenführen. Dachverbände wie der Internationale Deutschlehrerinnen- und Deutschlehrerverband/IDV wiederum können Mitglied in einem übergreifenden Dachverband sein, der eine Vernetzung von Verbänden bildet. Im Rahmen der Sprachverbände sind übergreifende Dachverbände normalerweise nicht nur auf eine einzelne Sprache ausgerichtet, sondern beziehen mehrere (Fremd)Sprachen ein.

Die Verbände, die uns im vorliegenden Rahmen und unter dem Blickwinkel der Deutschförderung interessieren, sind in der Regel ehrenamtlich wirkende Einrichtungen. Als Non-Profit-Organisationen (NPOs) setzen sie keinen wirtschaftlichen Gewinn an, nutzen vielmehr die eingehenden Finanzmittel ausschließlich für die Realisierung ihrer Verbandsziele zu Gunsten ihrer Mitglieder. Die erste Einnahmequelle gemeinnütziger Verbände bilden die Mitgliedsbeiträge, weshalb die ständigen Bemühungen um eine adäquate Mitgliederzahl von primärer Bedeutung sind. Neben der finanziellen Absicherung steht bei dieser Intention jedoch vor allem die Bestrebung im Mittelpunkt, durch eine möglichst hohe Mitgliederzahl die verbandspolitischen Ziele entsprechend wirkungsvoll nach innen umzusetzen wie gleichzeitig den Verband und seine Zielsetzungen überzeugend nach außen vertreten zu können. Mit der Wirkung nach außen ist etliches verbunden, worauf im Beitrag eingegangen werden soll. Zwei wichtige Punkte davon seien hier schon erwähnt: 1) Verbände möchten nicht nur für die eingeschriebenen Mitglieder sinnvoll wirken, sondern gleichzeitig immer auch für weitere Interessenten Ausstrahlungskraft ausüben; 2) Verbände, und dies gilt vor allem für Fachverbände, die ja gleichzeitig die zugehörigen Berufsstände mit einbeziehen, möchten in die Öffentlichkeit wirken und sich in die sprachenpolitischen Diskussionen im eigenen Land einbringen. Im Fall weltweit wirkender Verbände gilt dies auf internationaler Ebene.

Marianne Hepp, Universität Pisa, Italien, marianne.hepp@unipi.it

https://doi.org/10.1515/9783110479232-006 
Für das Erreichen ihrer Zielsetzungen suchen Fachverbände den Dialog mit den zuständigen staatlich-ministeriellen Einrichtungen. Im Falle der Fachverbände für Deutschlehrende, um die es in diesem Beitrag nun ganz konkret gehen soll, ist für das Erreichen ihrer wichtigsten Zielsetzungen die ständige Kooperation mit den für die Förderung und Unterstützung der deutschen Sprache und Kultur zuständigen Mittler- und Kulturorganisationen von grundlegender Bedeutung. Wichtig ist zudem die Vernetzung mit den institutionellen Bildungseinrichtungen Universität und Schule. Diese sind wichtige Orte für die Verbreitung der deutschen Sprache und Kultur weltweit, und sie sind gleichzeitig Arbeitgeber für die Deutschlehrenden. Hinzu kommen Momente der bedeutenden punktuellen Zusammenarbeit mit den (weiteren) Verbänden und den Institutionen ihres Fachs, beispielsweise bei der Werbung für Deutsch oder bei der Organisation von Tagungen. So wird etwa die alle vier Jahre stattfindende Internationale Deutschlehrerinnen- und Deutschlehrertagung/IDT, die weltweit größte Konferenz für Deutsch als Fremd- und Zweitsprache, zumeist - wenngleich nicht ausschließlich - von den Landesverbänden und einer Universität des ausrichtenden Landes gemeinsam ausgerichtet und vom Dachverband IDV begleitet.

Im Folgenden soll ein synthetischer Überblick über Verbände in den deutschsprachigen Ländern und auf der weltweiten Ebene vorgelegt werden, deren Zielsetzung die Förderung der deutschen Sprache und des Berufsstandes der Deutschlehrenden im internationalen Bereich ist (Abschnitt 2), gefolgt von Beispielen an sprachenpolitischen Momenten für die Deutschförderung durch Verbände (Abschnitt 3). Ein Fazit über die verbandspolitischen Handlungsmöglichkeiten (Abschnitt 4) rundet den Beitrag ab.

\section{Fachverbände für Deutsch als Fremd- und Zweitsprache}

Deutschlehrer- und Germanistenverbände sind „Berufsverbände, die durch ein durchgängiges berufliches Interesse ihrer Mitglieder zusammengehalten werden“ (Ammon 2015: 1132). Ihre gemeinsame verbandspolitische Zielsetzung ist die Förderung einer starken internationalen Stellung der deutschen Sprache. Eng mit dieser Zielsetzung verbunden ist „die Unterstützung der Deutschlehrenden in ihrer beruflichen Tätigkeit und fachlichen Aus- und Fortbildung“, wie dies beispielsweise der Internationale Deutschlehrerinnen- und Deutschlehrerverband e. V. (IDV) auf seiner Eingangsseite (http://idvnetz.org/was-ist-der-idv) und in seiner Satzung formuliert. 
Im Blickfeld des vorliegenden Beitrags stehen international vernetzte Verbände für Deutsch als Fremd- und Zweitsprache, die sowohl als fachliche Interessensvertretungen für ihre Mitglieder fungieren als auch „eine sprachenpolitische Vertretung dar[stellen], die die Positionierung des Faches [DaF/DaZ] und die internationale Stellung der deutschen Sprache beeinflussen “ (Hufeisen \& Sorger 2015: 166).

\subsection{Fachverbände in den deutschsprachigen Ländern}

Hinsichtlich der Bezeichnung „deutschsprachige Länder“ sei hier vorausgehend auf die landesgrenzenüberschreitende Verbreitung der deutschen Sprache hingewiesen, die unterschiedliche Definitionen für diesen Terminus ermöglicht (vgl. Ammon 2015: 154-157). In einem weiter gefassten Sinne sind damit die Länder mit Deutsch als nationaler und/oder regionaler Amtssprache gemeint, wobei es sich um insgesamt sieben Staaten handelt: neben Deutschland, Österreich, der Schweiz und Liechtenstein sind Luxemburg (Deutsch als nationale Amtssprache), Belgien und Italien (Deutsch als regionale Amtssprache) hierin eingeschlossen. In einem enger gefassten Sinne sind mit deutschsprachige Länder diejenigen Staaten gemeint, in denen Deutsch Amtssprache und gleichzeitig die Erstsprache der Bevölkerungsmehrheit ist. Dies ist für die ersten vier aufgelisteten Länder der Fall. In Luxemburg dagegen bekennt man sich mehrheitlich zu Letzeburgisch als Muttersprache, in Belgien und Italien wird Deutsch jeweils nur von einem kleinen Teil der Bevölkerung als Erstsprache gesprochen.

Alle Deutschlehrerverbände der deutschsprachigen Länder sind Mitglied im Dachverband IDV. Die einzige Ausnahme bildet Liechtenstein, das bisher noch keinen Landesverband für Deutsch gegründet hat, dabei aber gleichermaßen mit dem IDV vernetzt ist und bei den Unternehmungen des Dachverbands aktiv mitwirkt, vor allem bei der IDT. Mitglied im Dachverband IDV sind zudem fast alle Länder, in denen Deutsch Minderheitssprache (aber nicht staatliche Amtssprache) ist: Brasilien, Dänemark, Frankreich, Polen, Tschechien, Ungarn, Rumänien, Russland (s. Abschnitt 1.2). Bisher nicht vertreten ist Namibia, der einzige Staat, der mit einer deutschsprachigen Minderheit aus einer ehemaligen Kolonie Deutschlands hervorging. 
Im Folgenden werden diejenigen Fachverbände aus Deutschland, Österreich und der Schweiz kurz vorgestellt, die in Verbindung mit den jeweiligen staatlichen Einrichtungen wirken und international durch Mitgliedschaft im IDV besonders intensiv vernetzt sind. ${ }^{1}$

\subsubsection{Der Fachverband für Deutsch als Fremd- und Zweitsprache (FaDaF)}

Der am 7. September 1989 - kurz vor dem Mauerfall - gegründete Fachverband für Deutsch als Fremd- und Zweitsprache e. V. (FaDaF) sieht sich als „Interessenvertretung für DaF-/DaZ-Lernende, -Lehrende und -Institutionen“ (vgl. http:// www.fadaf.de/de/wir_ber_uns/der_fadaf/).

$\mathrm{Zu}$ den Zielsetzungen des FaDaF gehört die Förderung des interkulturellen Austausches zusammen mit dem Erlernen der deutschen Sprache. Der Fachverband mit seinen gegenwärtig ca. 740 Mitgliedern kümmert sich um die Belange der Fächer DaF und Daz und der in diesem Bereich tätigen Personen. Seine Programmschwerpunkte umfassen die Aus- und Fortbildung von Lehrkräften, die Förderung des wissenschaftlichen Nachwuchses für Deutsch als Fremd- und Zweitsprache und gute/bessere Rahmenbedingungen für ausländische Studierende und Wissenschaftler an deutschen Hochschulen. In der letzten Zeit setzt sich der FaDaF insbesondere für die sprachliche Integration von Geflüchteten und in diesem Zusammenhang auch für eine Verbesserung der Situation der Honorarkräfte in den öffentlich geförderten Integrationskursen ein.

Seit 2015 ist der FaDaF Mitglied im Gesamtverband Moderne Fremdsprachen (GMF) und gewährleistet dadurch die Kontaktpflege zu den weiteren Sprachverbänden in Deutschland.

Die FaDaF-Jahrestagung, die Publikationsreihe Materialien Deutsch als Fremd- und Zweitsprache und die Fachzeitschrift InfoDaF (herausgegeben in Zusammenarbeit mit dem DAAD) stellen den wissenschaftlichen Kern der facettenreichen FaDaF-Arbeit dar. Auch die Internet-Präsenz des Verbands ist hoch. Die Mitglieder wie auch weitere Interessenten erhalten Informationen zum Bereich der Verbandsarbeit samt Fachtagungen, Ergebnissen von Umfragen und Erhebungen (etwa zum Thema DaF- und Germanistikstudiengänge weltweit) usw. über die Mitgliederzeitung fadaf aktuell online.

1 Für einen Blick in die gesamte weitläufige Landschaft der nationalen Verbände, Vereine und Stiftungen, die sich ebenfalls verdienstvoll um die Förderung der deutschen Sprache kümmern, siehe Ammon (2015: 1124-1131). 
Als Aufsichtsorgan der Deutschen Sprachprüfung für den Hochschulzugang (DSH), welche die sprachliche Studierfähigkeit von nicht-muttersprachlichen StudienbewerberInnen feststellt, kommt dem FaDaF eine zentrale Mittlerposition zwischen Hochschulen, Prüfungsorganen und der Hochschulrektorenkonferenz $\mathrm{zu}$.

\subsubsection{Der Österreichische Verband für Deutsch als Fremdsprache/ Zweitsprache (ÖDaF)}

Der 1984 gegründete ÖDaF sieht sich ,als Netzwerk und Forum für seine Mitglieder und alle DaF/DaZ-Interessierten“ (http://www.oedaf.at/site/wirueberuns). Ein besonderes Anliegen sind dem rund 600 Mitglieder zählenden Fachverband die ständige Professionalisierung und Qualitätssicherung im DaF/DaZ-Unterricht, zusammen mit der Verbesserung der Rahmenbedingungen für Lehrende. Die breite Themenpalette des ÖDaF im Bereich der Didaktik und Methodik des Fremd- und Zweitsprachenunterrichts fokussiert insbesondere auf die Mehrsprachigkeit in verschiedenen Lernkontexten. An prominenter Stelle auf der Eingangsseite der ÖDaF-Website findet sich ein Zitat aus den Strobler Thesen zu einer österreichischen Sprachenpolitik ${ }^{2}$ : „Ziel jedes sprachenpolitischen Engagements ist die Förderung der Mehrsprachigkeit. Dies schließt die Durchsetzung einer Sprache auf Kosten anderer Sprachen aus“. Eine grundlegende Voraussetzung für die Verbandspolitik des ÖDaF bildet die Mehrsprachigkeit in einer multiethnischen Gesellschaft, zusammen mit der Plurizentrik der deutschen Sprache. Die Positionen des Verbandes werden regelmäßig in Stellungnahmen zu aktuellen Themen nach außen getragen.

Den wissenschaftlichen Publikationskern des ÖDaF stellen die zweimal jährlich erscheinenden ÖDaF-Mitteilungen dar, die neben Fachartikeln zu aktuellen Themen im DaF/DaZ-Bereich auch kritische Einschätzungen sprachenpolitischer Entwicklungen in Österreich und Europa enthalten und eine Plattform für den internationalen Erfahrungsaustausch bilden. Ein Fixpunkt sind zudem die jährlichen ÖDaF-Tagungen, bei denen sich WissenschaftlerInnen, Unterrichtende, Studierende und am Fach Interessierte über neueste Forschungsergebnisse infor-

2 Die 1994 vom Vorstand, von Mitgliedern und Freunden des ÖDaF erarbeiteten Strobler Thesen zur Sprachenpolitik sind das Ergebnis einer eingehenden Auseinandersetzung mit der österreichischen Sprachenpolitik und stellen die sprachenpolitischen Grundsätze des ÖDaF zusammen (www.oedaf.at/stroblerthesen). 
mieren und Erfahrungen austauschen können. Verbandspolitisch ziemlich einzigartig sind die ÖDaF-Stammtische, die regelmäßig in unterschiedlichen Städten Österreichs abgehalten werden und Interessenten vor Ort zu den unterschiedlichsten fachspezifischen Themen zusammenführen (http://www.oedaf.at/site/interessenvertretungsprac/stammtische).

Der Verband ist auch gut mit anderen Verbänden und Organisationen (z. B. Verband für Angewandte Linguistik, Netzwerk SprachenRechte) vernetzt und agiert gemeinsam mit diesen in verschiedenen sprachpolitischen Belangen. Als Verband eines deutschsprachigen Landes sind für den ÖDaF die Entwicklungen des Deutschen in anderen Ländern von größtem Interesse. Er arbeitet daher ständig mit vielen Deutschlehrendenverbänden weltweit zusammen.

\subsubsection{Der Arbeitskreis Deutsch als Fremdsprache - Deutsch als Zweitsprache in der Schweiz (AkDaF) und der Verein der Lehrenden in Deutsch als Fremd- und Zweitsprache (DaF/DaZ) an Hochschulen in der Schweiz (Ledafids)}

Deutsch ist in der Schweiz neben Französisch (22.5\%), Italienisch (8.1\%) und Rätoromanisch $(0,5 \%)$ eine der vier Landessprachen und eine der drei Amtssprachen. Mit 63,5 Prozent hat Deutsch den höchsten Sprecheranteil und ist in allen Landesteilen obligatorisches Schulfach: in der deutschsprachigen Schweiz als Schulsprache bzw. L1, in den französischsprachigen und zweisprachigen Regionen der Westschweiz als erste Fremdsprache ab dem 3. Primarschuljahr, im Tessin als zweite Fremdsprache ab der 7. Klasse. ${ }^{3}$

Die landesinterne und auswärtige Sprachenpolitik der beiden Schweizer Verbände spielt sich in einem Ausgangsrahmen der Mehrsprachigkeit im Lande ab, der teilweise zu anderen Gewichtungen führt, als dies für die Sprach- und Verbandspolitik der Nachbarländer Deutschland und Österreich der Fall ist. ${ }^{4}$ Die Landespolitik fördert hier die Gleichberechtigung aller vier Landessprachen, was vorwiegend nach innen, weitaus weniger nach außen geschieht. Dies bedeutet, dass es durch den außenpolitischen Fokus auf die Mehrsprachigkeit der Schweiz von staatlicher Seite kaum strukturelle Unterstützung für Projekte zur Sichtbarmachung und Verbreitung von Deutsch im internationalen Kontext gibt, wie

3 Berichtteil des AkDaF in Koglbauer \& Jarzabek (im Druck).

4 Vgl. dazu ausführlich Koglbauer \& Jarzabek (im Druck). 
gleichzeitig auch keine vergleichbaren Mittlerorganisationen ${ }^{5}$ existieren. Dies stellt die Fachverbände AkDaF und Ledafids vor besondere Herausforderungen im internationalen Handlungsfeld, etwa bei der Ausrichtung einer IDT (zuletzt 2017 in Freiburg i. Ü.). Als gewisses Gegengewicht zu der landesextern etwas zurückhaltenden Position könnte man die überaus positive Seite der landesinternen Situation für Deutsch im institutionellen Bereich erwähnen: Deutsch ist an öffentlichen Schulen in der Schweiz, wenn nicht Muttersprache, so überwiegend erste Fremdsprache, und Kenntnisse der Mehrheitssprache sind in vielen Berufen förderlich oder unabdingbar.

Der 1986 gegründete Arbeitskreis Deutsch als Fremdsprache - Deutsch als Zweitsprache/AkDaF (ca. 600 Mitglieder) und der 1988 entstandene Verein der Lehrenden für Deutsch als Fremd- und Zweitsprache an Hochschulen in der Schweiz/Ledafids (55 Mitglieder) setzen sich auf internationaler Ebene für die Wahrnehmung ihres Landes als Teil des deutschsprachigen Kultur- und Sprachraumes in Lehrmaterialien und für die Wahrnehmung von Deutsch als plurizentrische Sprache ein. Der AkDaF (http://www.akdaf.ch/) engagiert sich im Wissensaustausch und für die Weiterbildung seiner Mitglieder etwa durch einen eigenen, im Privatschulbereich anerkannten Weiterbildungslehrgang (IDIconTOTO) und die Durchführung einer Jahrestagung zu aktuellen DaF/DaZ bezogenen Themen. Die Information an die Mitglieder erfolgt über den „AkDaF Rundbrief“. Seit 2016 hat der Fachverband mit einer Vertreterin Einsitz im Schweizerischen Verband für Weiterbildung (SVEB) und hofft dadurch, künftig noch stärker auf (sprachen-)politischer Ebene Einfluss auf die Erwachsenenbildung nehmen zu können.

Der Ledafids versteht sich als Forum für den wissenschaftlichen Austausch und für fachbezogene Weiterbildung (https://www.ledafids.ch/) und führt einmal jährlich eine zweitägige geschlossene Mitgliedertagung durch, bei der aktuelle, den eigenen Arbeitsbereich betreffende Themen offen diskutiert und auf den Prüfstand gestellt werden. Seit 2006 organisieren die beiden Verbände gemeinsam eine alle zwei Jahre stattfindende DaF/DaZ-Tagung in Bern (http://www.dafdaztagung.ch/).

5 Die offizielle Politik in Deutschland und Österreich delegiert die auswärtige Vermittlung von Kultur und Sprache an verschiedene Mittlerorganisationen, die zwar zum größten Teil mit staatlichen Mitteln finanziert werden, inhaltlich aber weitestgehend selbständig arbeiten können (vgl. Sorger 2012: 171). Die größten Mittlerorganisationen für die Unterstützung der Fachverbände für Deutsch sind das Goethe-Institut (D) und das Österreich Institut (A). 
Allen hier erwähnten Verbänden der deutschsprachigen Länder ist in erster Linie ihr internationales Engagement für die Förderung von Deutsch (als plurizentrische Sprache) gemeinsam. Als Mitglieder im internationalen Dachverband IDV, für den sie regelmäßig auch die ExpertInnen D-A-CH im Vorstandsgremium stellen, gewährleisten sie in festgelegter Abwechslung, nach dem sogenannten Rotationsprinzip D-A-CH, die Ausrichtung der Internationalen Tagung für Deutschlehrerinnen und Deutschlehrer/IDT als der weltweit größten Fachkonferenz für Daf/DaZ in ihrem jeweiligen Land. Als feste Vertretungen im DACHL-Gremium des IDV (ehem. DACHL-Arbeitsgruppe) unterstützen sie die Umsetzung des DACH-Prinzips, d. h. die fachlich-didaktische Berücksichtigung der Plurizentrik und Plurikulturalität der deutschen Sprache (zur Plurizentrik vgl. ausführlich Ammon 1995) auf internationaler Ebene (http://idvnetz.org/dachl-online).

\subsection{Fachverbände weltweit}

Nicht nur im deutschsprachigen Raum, sondern auch weltweit fördern Fachverbände die Vermittlung der deutschen Sprache und die Kultur der deutschsprachigen Länder. Im Folgenden wird das Augenmerk auf diejenigen Verbände gerichtet, die - zusammen mit den in Abschnitt 1.1 genannten Verbänden - im Dachverband IDV, dem Internationalen Deutschlehrerinnen- und Deutschlehrerverband e. V., durch Mitgliedschaft vernetzt sind. Es handelt sich dabei überwiegend um einzelne nationale Deutschlehrer- und Germanistenverbände, unter denen sich aber auch Dachverbände und Deutschsektionen von multilingualen Vereinigungen befinden. Gegenwärtig sind im IDV insgesamt 94 Verbände aus 85 Ländern aller Kontinente vernetzt, wie die folgende Tabelle aufzeigt:

Tab. 1: Die nationalen Deutschlehrer- und Germanistenverbände des Internationalen Deutschlehrerinnen- und Deutschlehrerverbands e.V./IDV, mit Einzel- und Dachverbänden (letztere fettgedruckt), Stand 2018 (http://idvnetz.org/verbande-weltweit)

\begin{tabular}{llll}
\hline Ägypten & Frankreich & Litauen & Schweden \\
Albanien & Georgien & Luxemburg & Schweiz (2) \\
Algerien & Ghana & Madagaskar & Senegal \\
Argentinien & Griechenland & Malaysia & Serbien \\
Armenien & Großbritannien & Mali & Slowakei \\
Australien & Guatemala & Marokko & Slowenien \\
Belarus & Indien & Mazedonien & Spanien \\
Belgien & Indonesien & Mexiko & Südafrika \\
\hline
\end{tabular}




\begin{tabular}{llll}
\hline Benin & Irland & Moldau & Südkorea \\
Bolivien & Island & Mongolei & Taiwan \\
Bosnien-Herzegowina & Italien (2) & Neuseeland & Thailand \\
Brasilien & Japan & Niederlande & Togo \\
Bulgarien & Kamerun & Nigeria & Tschechien \\
Burkina Faso & Kanada & Norwegen & Tunesien \\
Chile & Kasachstan & Österreich & Türkei \\
Côte d'Ivoire & Kirgistan & Paraguay & Ukraine (2) \\
Dänemark (5) & Kolumbien & Peru & Ungarn \\
Deutschland & Kosovo & Polen & Uruguay \\
Ecuador & Kroatien & Portugal & USA \\
Estland & Kuba & Rumänien & Usbekistan \\
Finnland & Lettland & Russische Föderation & Venezuela \\
& & (3, darunter ein Dach- & Vietnam \\
& & verband) & \\
\hline
\end{tabular}

Die sich darunter befindenden fünf Dachverbände (fett gedruckt) haben wiederum die folgende Anzahl an einzelnen Deutschlehrerverbänden:

Tab. 2: Dachverbände als regionale Zusammenschlüsse von Deutschlehrerverbänden im Internationalen Deutschlehrerinnen- und Deutschlehrerverband e.V./IDV, Stand 2018 (http://idvnetz.org/verbande-weltweit)

\begin{tabular}{lll}
\hline $\begin{array}{l}\text { Bezeichnung } \\
\text { des nationalen Dachverbands }\end{array}$ & $\begin{array}{l}\text { Gesamtzahl } \\
\text { der Mitgliedsverbände }\end{array}$ & Gesamtzahl der Mitglieder \\
\hline $\begin{array}{l}\text { ABraPA - Brasilianischer Deutsch- } \\
\text { lehrerverband }\end{array}$ & 7 & 870 \\
$\begin{array}{lll}\text { DLV Indonesiens } & 21 \text { (Zahl nach Orten/Re- } \\
\text { gionen Indonesiens) }\end{array}$ & 400 \\
$\begin{array}{l}\text { F.A.G.E - Federación de Asociaciones } \\
\text { de Germanistas en España/Germanis- }\end{array}$ & 9 & 490 \\
$\begin{array}{l}\text { ten- und Deutschlehrerverband Spani- } \\
\text { ens }\end{array}$ & \\
$\begin{array}{l}\text { Kanadischer Deutschlehrerverband } \\
\begin{array}{l}\text { Überregionaler DLV Russlands } \\
\hline\end{array}\end{array}$ & 9 & 500 \\
\hline
\end{tabular}


Berücksichtigt man auch diese Einzelzusammensetzung, so beträgt die Zahl der IDV-Mitgliedsverbände gegenwärtig (2018) 146, während diejenige der Einzelmitglieder aus allen IDV-Verbänden mit rund 30.000 beziffert werden kann. Mindestens genauso wichtig wie jegliche Auszählung, die unabdinglich immer wieder leicht variieren muss, ist die Feststellung einer kontinuierlich steigenden Tendenz für Verbandsmitgliedschaft und Vernetzung: So haben sich für die kommende Vertreterversammlung 2019 in Leipzig drei weitere Verbände für eine Aufnahme in den Dachverband gemeldet. Seit Gründung des IDV im Jahr 1968 hat sich die Zahl seiner Mitglieder stetig erhöht, im Schnitt handelt es sich dabei um zwei Verbände pro Vertreterversammlung, die im Zwei-Jahresrhythmus abgehalten wird und über die Anträge abstimmt. Die Zahl der Austritte, meist durch Auflösung des nationalen Verbands, ist dagegen sehr gering.

Es würde den Rahmen dieses Beitrags sprengen, alle 94 Mitgliedsverbände vorzustellen. Da sich ihre sprachenpolitischen Zielsetzungen in vielen Fällen mit denjenigen des Dachverbands überschneiden, soll nun zuerst der IDV präsentiert werden. Danach, im anschließenden zweiten Teil, wird punktuell auf die Mitgliedsverbände Bezug genommen. Berücksichtig wird dabei auch eine Umfrage, die der IDV-Vorstand in den Jahren 2016-2017 zum Thema „Sprachenpolitisches Handeln von Verbänden“ durchgeführt hat. ${ }^{6}$

\subsection{Der IDV als Dachverband für Deutsch als Fremd- und Zweitsprachenverbände}

Der Internationale Deutschlehrerinnen- und Deutschlehrerverband e. V. IDV ${ }^{7}$ fungiert als weltweites Netzwerk seiner Mitgliederverbände für ein breites Spektrum von Aufgaben und Aktivitäten für DaF und DaZ. ${ }^{8}$ Eine erste Zielsetzung ist die Unterstützung der Deutschlehrenden in ihrer beruflichen Tätigkeit und fach-

\footnotetext{
6 Die Ergebnisse der Umfrage wurden bei der IDT 2017 in Freiburg/CH vorgestellt, im Rahmen eines Workshops der Zielgruppenspezifischen Arbeitsgruppe /SIG 2.4 und gingen in die sprachenpolitische Resolution der IDT

(https://www.idt-2017.ch/docs/resolution/Freiburger_Resolution_IDT_2017_komplett.pdf) ein. Vgl. Koglbauer \& Jarzabek (im Druck).

7 Für alle folgenden Angaben zum IDV auf der Website siehe: www.idvnetz.org.

8 Seit Oktober 2017 hat der IDV einen festen Verbandssitz bei interDaF am Herder Institut der Universität Leipzig, abgestimmt durch die IDV-Vertreterversammlung im Rahmen der XVI. IDT in Freiburg/Schweiz. Zuvor wurde der Verbandssitz abwechselnd im jeweiligen Land der amtierenden PräsidentInnen eingerichtet.
} 
lichen Aus- und Fortbildung. Weitere vorrangige Zielsetzungen sind die Mitwirkung bei der Weiterentwicklung des Fachs DaF/DaZ und der sprachenpolitischen Unterstützung der Stellung der deutschen Sprache in der Welt. Diese Unterstützung soll im Rahmen der Mehrsprachigkeit erfolgen, als angemessene Positionierung von DaF/DaZ im Kontext einer erweiterten Fremdsprachenvermittlung - in der Einsicht, dass nur eine ausdifferenzierte Sprachenkenntnis die Erschließung von vielfältigen Sprachkulturen ermöglicht (s. 2.1). Eine grundlegende sprachenpolitische Zielsetzung für den IDV ist gleichzeitig die Vermittlung der deutschen Sprache als plurizentrisches Kommunikationsmittel (s. 2.2).

Der IDV erreicht seine sprachenpolitischen Vereinsziele der Weiterbildung und Vernetzung seiner Mitglieder vor allem durch die Ausrichtung großer Fachkonferenzen und weiterer einschlägiger Veranstaltungen. An erster Stelle ist dabei die Internationale Deutschlehrerinnen- und Deutschlehrertagung zu nennen (IDT), gefolgt von der alle zwei Jahre stattfindenden Internationalen Deutscholympiade (IDO), die von Mitgliedsverbänden des IDV in Südosteuropa als nationale Deutscholympiade(n) konzipiert wurde und heute vom IDV gemeinsam mit dem Goethe-Institut ausgeführt wird. Bei der IDO 2018 in Freiburg i. Br. treffen über 140 Jugendliche aus 75 Ländern zusammen, das sind zehn Länder mehr als bei der vorhergehenden IDO 2016 in Berlin. In den IDO-Wettbewerben werden nicht nur die Sprachkenntnisse, sondern genauso die interkulturelle Kompetenz, Teamfähigkeit und Kreativität der Jugendlichen bewertet.

Ein besonderer Preis bei der IDO ist der Fairnesspreis, der die Zielsetzung der Toleranz und des Miteinanders unterstreicht.

Bedeutend sind auch die zahlreichen Regionaltagungen (z. B. 2017 in Antigua/Guatemala für die zentralamerikanischen Verbände, 2018 in New Delhi für Südasien). Da die Bedingungen und Bedürfnisse der Deutschunterrichtenden in der Welt sehr unterschiedlich sind, bieten Regionaltagungen auf besondere Weise die Möglichkeit, Erfahrungen auszutauschen, Projekte zu entwickeln und Lösungen für gemeinsame Bedürfnisse in der Region zu erarbeiten. Nicht zuletzt können hier weitere Mitgliedsverbände aus der Region für die Zusammenarbeit im IDV gewonnen werden.

Zur Illustration der zahlreichen Projekte des IDV sollen an dieser Stelle zwei aktuelle Beispiele herausgegriffen werden: Ein Gemeinschaftsprojekt des IDV und des Goethe-Instituts trägt den Titel „Verbandsarbeit erfolgreich gestalten“. Aus einer Reihe von Workshops, die in unterschiedlichen Regionen der Welt zusammen mit Mitgliedsverbänden durchgeführt wurden, sind die „Handreichungen - Verbandsarbeit erfolgreich gestalten“ entstanden, die online für all diejenigen zur Verfügung stehen, die sich in die Arbeit von Verbänden einbringen oder deren sprachenpolitische Tätigkeit weiterentwickeln wollen. Ein weiteres 
IDV-Projekt, die Entwicklung einer Online-Plattform für Projekte der Verbände, will allen Mitgliedern die Gelegenheit geben, ihre kreative Arbeit öffentlich vorzustellen und sich gegenseitig in Projekten zu vernetzen.

Über seine Veranstaltungen und die Initiativen seiner Mitglieder berichtet der IDV in seinen verschiedenen Publikationsmedien, die online über die Website abrufbar und der allgemeinen Öffentlichkeit zugänglich sind: Dazu gehören das zweimal jährlich erscheinende IDV-Magazin, die regelmäßigen Mitteilungsseiten über die Vorstandsarbeit, IDV aktuell, sowie Neuigkeiten auf Facebook. Die nicht-öffentliche Kommunikation mit den Mitgliedern verläuft über Rundmails, über die auch die Protokolle der Vorstandssitzungen versandt werden. ${ }^{9}$

\section{Sprachenpolitik als Basis für die Verbandsarbeit}

Inwiefern ist Verbandsarbeit sprachenpolitisch, wie gliedert sie sich in die allgemeine Sprachenpolitik ein? Um darauf eine Antwort zu suchen, sei erst die Bedeutungsweite dieses Begriffs synthetisch dargestellt.

Nach Krumm (2007: 298) ist Sprachenpolitik die Bezeichnung sowohl für offizielle und staatliche Regelungen, die den Gebrauch, die Verbreitung und allgemein die Förderung von bestimmten Sprachen betreffen, als auch für alle Bemühungen um die Verwendung bestimmter Sprachen von Seiten nicht-staatlicher Einrichtungen, d. h. von Sprachlehrenden, JournalistInnen, FachexpertInnen usw. Sprachenpolitik fächert sich zuvorderst in zwei Hauptbereiche auf:

1) Sprachenpolitik nach innen (z. B. Festlegung des Sprachangebots im Bildungswesen eines Landes, Positionierung des Zweitsprachenunterrichts, gegenwärtig vor allem Integrationssprachkurse für Geflüchtete und Neubürger, Förderung von Minderheitensprachen im Land)

2) Sprachenpolitik nach außen (z. B. Förderung einer Landessprache außerhalb der Region, in der sie Amts- und Muttersprache ist; die erklärte Zielsetzung

\footnotetext{
9 Die Leitung des IDV wird von einem fünfköpfigen Vorstand ausgeführt, der auf vier Jahre gewählt wird (die maximale Amtszeit der einzelnen Vorstandmitglieder umfasst drei Kandidaturen, d. h. insgesamt 12 Jahre). Dem Kernvorstand stehen ExpertInnen aus den deutschsprachigen Ländern (ohne Stimmrecht) zur Seite, wahlweise auch weitere kooptierte Mitwirkende. Aus den Verbänden der deutschsprachigen Länder (Abschnitt 1) dürfen keine Vorstandmitglieder gewählt werden. Bedingung für eine Kandidatur in das Gremium ist ein fester erster Wohnort in einem Land außerhalb D-A-CH-L.
} 
der Europäischen Union, jeder europäische Bürger solle bereits während der Schulzeit mindestens zwei moderne Fremdsprachen lernen).

Die Sprachenpolitik der Fachverbände für Deutsch als Fremd- und Zweitsprache ist vor allem mit dem zweiten Hauptbereich verbunden, mit der Sprachverbreitungspolitik. Dies gilt für den IDV als auf der internationalen Ebene wirkender Dachverband wie für alle seine Mitgliedsverbände. Eine gewisse Sonderstellung nehmen allerdings die Verbände der deutschsprachigen Länder ein (s. Abschnitt 1), die sich sowohl für die Förderung des Deutsch-als Zweitspracheunterrichts in ihren Ländern als auch für diejenige von Deutsch-als-Fremdsprachenunterricht auf der internationalen Ebene einsetzen.

\subsection{Deutschförderung im Hinblick auf Mehrsprachigkeit}

Die Vermittlung von Sprachen erfolgt in einer mehrsprachigen, sich ständig näher rückenden Welt. Nur eine umfassende Kenntnis (über) mehrere(r) Sprachen ermöglicht eine intensive Erschließung von und Begegnung mit vielfältigen Sprachkulturen. Als ein übergeordnetes sprachenpolitisches Ziel von Sprachverbänden muss daher die angemessene Positionierung der von ihnen geförderten Sprache(n) und Sprachkulturen im Kontext einer weiten, nicht einer engen, Fremdsprachenvermittlung gesetzt werden.

Der Dachverband IDV engagiert sich von Anfang seines Bestehens an für einen Fremdsprachenunterricht, der sich nicht in Konkurrenz $\mathrm{zu}$ anderen Sprachen sieht, vielmehr im Kontext der Mehrsprachigkeit agiert, als eine der vielen Stimmen in einem harmonischen Konzert der Sprachen. Diese Einstellung wird schon im Leitbild des Dachverbands deutlich ${ }^{10}$ :

In einer mehrsprachigen Welt kann das Lernen von Sprachen, mithin auch der deutschen, Menschen einander näher bringen. Das trägt zum gegenseitigen Verständnis bei und stärkt den Respekt vor der Würde aller Menschen, ihren vielfältigen Sprachen und Kulturen.

Der IDV unterstreicht seinen Mehrsprachigkeitsansatz nicht zuletzt auch durch seine Mitgliedschaft und intensive Zusammenarbeit in der Fédération Internationale des Professeurs de Langue Vivantes /FIPLV, dem weltweiten Dachverband für Fremdsprachenverbände. ${ }^{11}$ In diesem multilingualen Weltverband, aus dem

10 vgl. http://idvnetz.org/der-idv.

11 vgl. https://fiplv.com/. 
der IDV ursprünglich entstanden ist (vgl. Sorger 2012: 130-132), werden bei Kongressen und Sitzungen unterschiedliche Sprachen verwendet, wobei oft das System der rezeptiven Mehrsprachigkeit angewandt wird (jede/r Anwesende spricht seine/ihre Muttersprache und versteht die anderen Sprachen, ohne Einsatz eines Dolmetschers). Zu den wichtigsten Aufgaben der FIPLV gehört die Ausrichtung eines mehrsprachigen Weltkongresses, der alle drei Jahre abwechselnd in einer anderen Weltregion stattfindet. Darüber hinaus finden jährlich Regionaltagungen unter der FIPLV-Flagge weltweit statt. Bei diesen internationalen mehrsprachigen Kongressen wird seit einigen Jahren eine deutschsprachige Sektion abgehalten, geleitet von einem Vorstandsmitglied des IDV ${ }^{12}$. Auf diese Weise ergibt sich auch eine wirkungsvolle Ergänzung zu den bewusst einsprachig gehaltenen Kongressen des IDV, bei denen andere Zielsetzungen im Vordergrund stehen, darunter in erster Linie auch die Förderung der deutschen Wissenschaftssprache. Dasselbe gilt für die alle fünf Jahre abgehaltenen Kongresse der Internationalen Vereinigung für Germanistik /IVG ${ }^{13}$. Bei den Tagungen des IDV wie der IVG ist festzustellen, dass die Sektionen zum Thema Mehrsprachigkeit zunehmen, dass also großes Interesse der Mitglieder an diesem Thema vorhanden ist.

\subsection{Deutschförderung unter Einbezug des DACH-Prinzips}

Die Bemühungen um ein weltweites Wahrnehmen und didaktisches Umsetzen der sprachlich-kulturellen Vielfalt des deutschen Sprachraums war von Anfang an ein wichtiges Ziel im IDV ${ }^{14}$. Nicht zuletzt auch deshalb, weil schon bei seiner Gründung am 8. April 1968 in Zagreb VertreterInnen aus den damals vier deutschsprachigen Ländern (BRD, DDR, A, CH) und aus Luxemburg anwesend waren (vgl. Sorger 2012: 139). Einen entscheidenden Aufschwung erfuhr die Förderung des DACH-Prinzips jedoch erst durch die Zusammenstellung der ABCDThesen (1990) ${ }^{15}$ und die (Neu-)Einrichtung einer DACHL-Arbeitsgruppe (2007,

\footnotetext{
12 Vgl. die Nordisch-Baltische Regionaltagung 2016 in Tallinn/Estland, deren Ausrichter der Estnische Dachverband der Fremdsprachenlehrer/innen EVÕL war, sowie um die I. Osteuropäische Regionaltagung des FIPLV 2017 in Varna/Bulgarien (Konstantin-Preslavski-Universität Schumen), organisiert vom Bulgarischen Verband der Englischlehrer (BETA). Vgl. De Carvalho \& Lamb (in Vorbereitung).

13 Der nächste IVG-Kongress findet 2020 in Palermo statt (vgl. http://ivg2020.unipa.it/), die nächste IDT 2021 in Wien (http://www.idt-2021.at/).

$14 \mathrm{Zu}$ allen DACHL-Initiativen des IDV vgl. http://idvnetz.org/dachl-online.

15 Erschienen u.a. im IDV-Rundbrief 1990/45, S. 15-18 (www.idvnetz.org/publikationen/rundbrief/rb45.pdf). Diese Thesen forderten eine Landeskunde im Fremdsprachenunterricht, die sich
} 
2018 institutionalisiert als DACHL-Gremium des IDV), verbunden u.a. mit der Durchführung von DACHL-Landeskunde-Seminaren, Wettbewerben, der Publikation von Fachbeiträgen (z.B. Demmig, Hägi \& Schweiger 2013), der Durchführung von Fachtagungen (2011 in Frauenchiemsee, 2018 in München) und der Formulierung des DACH-Prinzips:

Das DACH-Prinzip geht von der grundsätzlichen Anerkennung der Vielfalt des deutschsprachigen Raumes im Rahmen des Unterrichts der deutschen Sprache, der Vermittlung von Landeskunde, der Produktion von Lehrmaterialien sowie der Aus- und Fortbildung von Unterrichtenden aus. In der Umsetzung bedeutet dies - im gemeinsamen Bemühen um die Stützung und Förderung des Deutschunterrichts weltweit - die gleichwertige Einbeziehung der unterschiedlichen sprachlichen und landeskundlichen Dimensionen des deutschsprachigen Raumes im Rahmen wissenschaftlicher Arbeiten, in Aktivitäten der Mittlerorganisationen, in der Entwicklung von Konzepten und Projekten sowie in allen Formen der Präsentation fachlicher Aktivitäten, vor allem aber auch in der Praxis des Unterrichts. [...] (Demmig, Hägi \& Schweiger 2013: 11-12).

Ziel des DACH-Prinzips ist es, „im Kontext aktueller gesellschaftlicher Herausforderungen und Veränderungen durch gemeinsame Projekte, institutionalisierte Kooperation und das Nutzen von Synergien den Deutschunterricht weltweit zu stützen und zu fördern.“ (Shafer \& Baumgartner (im Druck)). Es kann hier wohl erwähnt werden, dass insbesondere durch die ständigen Bemühungen des IDV, verbunden mit denjenigen der IDT-Ausrichtenden und den Mitwirkenden bei den unterschiedlichen Fortbildungsaktivitäten, zahlreiche Verbände und Deutschlehrende weltweit die kulturelle und linguistische Gleichwertigkeit aller Länder und Regionen des amtlich deutschsprachigen Raums systematisch und zeitgemäß vermitteln.

Viele Mitgliedsverbände halten in ihren Ländern und Regionen DACHL-Tagungen ab und verweisen auf ihren Websites auf die Plurizentrik des Deutschen. ${ }^{16}$

nicht auf Staaten- und Institutionenkunde reduziere, vielmehr durch Einsatz vielfältiger Materialien, gleichzeitig bereichert durch die eigene Erfahrung der Unterrichtenden, alle deutschsprachigen Regionen lebendig werden lasse. Hier klingt auch schon das Konzept der „erlebten Landeskunde" an, das einige Jahre später die Basis für die DACHL-Seminare bilden sollte.

16 Als ein außereuropäisches Beispiel sei der Mexikanische Deutschlehrerverband genannt, der auf seiner Website das DACH-Prinzip vorstellt und „Ideen, Texte über DACHL und Plurizentrik, Fotos, Musik, Filme, Unterrichtsmaterial usw.“ sammelt

(http://www.ampal.org/index.php/de/). 


\subsection{Deutschförderung durch Mitgestaltung der Deutschlehrer- bildung}

Guter Deutschunterricht braucht gute Deutschlehrpersonen, was wiederum eine gute Aus- und Weiterbildung voraussetzt und damit ein weltweites Desiderat/Interesse darstellt. Auch steigt der Bedarf an Deutschlehrkräften in vielen Regionen der Welt, z. B. Indien und China, weiterhin an. Eine institutionelle Lehrerausbildung ist aber auch gegenwärtig noch längst nicht überall eine Selbstverständlichkeit. Vielmehr werden an Stelle von umfassenden, speziell für die künftigen Deutschlehrer konzipierten Studiengängen in vielen Ländern, darunter auch europäischen, bestenfalls vereinzelte Module zur Einführung in die Didaktik und Methodik von DaF angeboten.

Schon aus diesem Grund muss die Qualifikation für den Lehrerberuf durch eine fundierte fachdidaktische und methodische Lehrerfortbildung gewährleistet werden. Fortbildungsangebote für Deutschlehrende nehmen in der Tat seit Jahren $\mathrm{zu}$, sie werden online und in Kursen vor Ort an verschiedenen Schulen und Universitäten weltweit durchgeführt.

Die Fachverbände für Deutsch bringen sich in die Fortbildung für Deutschlehrende intensiv mit ein, indem sie an ihren Fachtagungen und über ihre Publikationsmedien die am Thema Interessierten über den neuesten Stand der Forschung und Didaktik informieren.

Vor allem sind es die Kongresse der Fachverbände, die alle direkt oder indirekt die Fortbildung von Deutschlehrenden zum Ziel haben. Da sie meist eine Brücke zwischen Theorie und Praxis bilden, kommen sie auch dem Bedürfnis nach mehr Praxisorientiertheit entgegen, das sich durch das vielerorts fehlende Referendariat oder eine ausbleibende Hospitation und Unterrichtserprobung ergibt. Insbesondere die IDTs vermögen die Kluft zwischen einem rein fachphilologisch ausgerichteten Studium und unmittelbar darauf anschließender Lehrtätigkeit zu überbrücken. Sie verbinden unter einem jeweiligen Kongressthema Fachvorträge von DaF- und DaZ-SpezialistInnen und Vorträge in den zahlreichen Sektionen (als ,Herz' der IDT), in denen Deutschlehrende an Schule und Universität über den Globus hinweg ihre eigenen Erfahrungen einbringen. Sie bieten zum selben Rahmenthema didaktische Werkschauen an, die Einblick in den Lehralltag gewährleisten und setzen die Diskussion in sprachpolitischen Podien fort, zu denen auch Entscheidungsträger aus Politik und Wirtschaft eingeladen werden.

In einer der elf Thesen der Freiburger Resolution zur Sprachenpolitik, die im Vorfeld der IDT 2017 von ebenso vielen thematischen Arbeitsgruppen zu Brennpunkten von DaF und DaZ erarbeitet wurden, wird in der These 9 auf das Thema 
Aus-, Fort- und Weiterbildung von Lehrenden Bezug genommen, indem folgende Forderung formuliert wird:

Die Ausbildung von Lehrkräften für DaF und DaZ sollte den Prinzipien Kompetenzorientierung und Berufsorientierung folgen. Fachwissenschaftliche Inhalte sind möglichst von Anfang an mit Fachdidaktik und Unterrichtspraxis zu verbinden, da Unterrichten im Spannungsfeld von fachdidaktischer Theorie, Erfahrungswissen von (angehenden) Lehrkräften, begleitendem Unterrichten und Reflexion von Unterricht erlernt wird. (https://www.idt2017.ch/docs/resolution/Freiburger_Resolution_IDT_2017_komplett.pdf)

\section{Ausblick}

Wie der vorliegende Beitrag aufzuzeigen versuchte, leisten Fachverbände für Deutsch weltweit in ehrenamtlicher Arbeit enorm viel für die Förderung der deutschen Sprache und die berufliche Vernetzung und ständige Fort- und Weiterbildung ihrer Mitglieder. Bei allen Erfolgen, beim erfreulichen Trend der steigenden Mitgliedschaften und neuen Verbandsgründungen, sind der Verbandsarbeit jedoch bestimmte Grenzen gesetzt, die es nach Möglichkeit zu überwinden gilt. Als erste Begrenzung kann der insgesamt zu gering bleibende Einfluss der Fachverbände auf die Bildungspolitik angesehen werden, der trotz einzelner Erfolgsmeldungen, z.B. in der Umfrage des IDV (vgl. Koglbauer \& Jarzabek im Druck), immer wieder von Verbandsmitgliedern beklagt wird. Auch die Situation des unzeitgemäßen „monolingualen Habitus“ (Gogolin 2008) in den Schulen dauert weiterhin an. Auf die Schwierigkeiten der Verbände, dies zu ändern, weist erneut vor kurzem ein bedeutender ehemaliger Präsident des IDV, Waldemar Pfeiffer, hin: „Versuche internationaler Fremdsprachenverbände, diesen Umstand [der Monolingualität, M.H.] zu ändern oder nur zu mildern, haben bis jetzt keinen spektakulären Umbruch gebracht“ (Pfeiffer 2015: 14). Das propagierte europaweite Bildungsmodell 1+2, das das obligatorische Lernen der Muttersprache plus zwei anderen Sprachen voraussetzt, ${ }^{17}$ sollte jedoch dauerhaft in die Unterrichtspraxis umgesetzt werden, nicht zuletzt auch, weil das Lernen von Sprachen, somit auch der deutschen Sprache, auf das Engste mit einer positiver Haltung zu anderen Kulturen und besserer Erkenntnis der eigenen in Verbindung stehen.

17 Vgl. Europäisches Parlament, Sprachenpolitik, in «Europäisches Parlament Service» (2017), http://www.europarl.europa.eu/factsheets/de/sheet/142/sprachenpolitik. 
Und für Fachverbände bedauerlich sind auch die nicht in allen Ländern angemessenen Entlohnungen für Deutschlehrende an Schule und Universität, wie teilweise auch im privaten Bereich.

Hier wäre eine nachhaltigere politische Unterstützung nötig, zusammen mit einer intensiveren direkten Zusammenarbeit zwischen Fachverbänden und sprachpolitischen Entscheidungsträgern. Ein systematischerer Einbezug der Fachverbände in die staatliche Sprachenpolitik unterschiedlicher Länder und in Kommissionen für die institutionellen Bildungspläne würde große Früchte auf internationaler Ebene tragen und nicht zuletzt den Deutschschülerinnen und Deutschschülern - als künftig politisch mitbestimmende und teilhabende MitbürgerInnen - zugute kommen.

\section{Literaturverzeichnis}

ABCD-Gruppe (1990): ABCD-Thesen zur Rolle der Landeskunde im Deutschunterricht. IDVRundbrief 45, 15-18.

Ammon, Ulrich (1995): Die deutsche Sprache in Deutschland, Österreich und der Schweiz. Das Problem der nationalen Varietäten. Berlin, New York: de Gruyter.

Ammon, Ulrich (2015): Die Stellung der deutschen Sprache in der Welt. Berlin u.a.: de Gruyter. Arbeitskreis DaF/DaZ in der Schweiz: http://www.akdaf.ch. Gemeinsame Jahrestagung der beiden Schweizer Verbände: http://www.dafdaztagung.ch/ (30.06.2018).

De Carvalho, Geraldo \& Terry Lamb (in Vorbereitung): IDV und FIPLV: Zwei internationale Dachverbände in 50-jähriger Vernetzung. IDV-Magazin 94/2018.

Demmig, Silvia, Sara Hägi \& Hannes Schweiger (Hrsg.) (2013): DACHL-Landeskunde. Theorie Geschichte - Praxis. München, ludicium.

Europäisches Parlament (Sprachenpolitik): http://www.europarl.europa.eu/factsheets/de/sheet/142/sprachenpolitik (30.06.2018).

Fachverband Deutsch als Fremd- und Zweitsprache/FaDaF: http://www.fadaf.de/. Leitlinien des FaDaF: http://www.fadaf.de/de/wir_ber_uns/der_fadaf (30.06.2018).

Fédération Internationale des Professeurs de Langues Vivantes: http://fiplv.org (30.06.2018). Gogolin, Ingrid (2008): Der monolinguale Habitus der multilingualen Schule. Münster: Waxmann.

Hufeisen, Britta \& Brigitte Sorger (2015): Die internationale Institutionalisierung von Deutsch als Zweit- und Fremdsprache. In Hans-Jürgen Krumm, Christian Fandrych, Britta Hufeisen \& Claudia Riemer (Hrsg.), Deutsch als Fremd- und Zweitsprache. Ein internationales Handbuch. 2 Halbbde., 166-172. Berlin, New York: de Gruyter Mouton.

IDT 2017: https://www.idt-2017.ch/. Freiburger Resolution zur Sprachenpolitik. 11 Thesen zur Stärkung und Weiterentwicklung von Deutsch als Fremd- und Zweitsprache. https://www.idt-2017.ch/docs/resolution/Freiburger_Resolution_IDT_2017_komplett.pdf (30.06.2018).

IDT 2021: http://www.idt-2021.at/ (30.06.2018). 
IDV \& Goethe Institut (2017): Handreichungen - Verbandsarbeit erfolgreich gestalten. Ein Gemeinschaftsprojekt. http://idvnetz.org/handreichungen-verbandsarbeit-erfolgreich-gestalten (30.04.2018).

Internationaler Deutschlehrerinnen- und Deutschlehrerverband e.V./IDV: www.idvnetz.org. Leitlinien des IDV: http://idvnetz.org/was-ist-der-idv. Dachl-Website des IDV: http://idvnetz.org/dachl-online. Internationale Mitgliedsverbände des IDV: http://idvnetz.org/verbande-weltweit (30.06.2018).

IVG 2020: http://ivg2020.unipa.it/ (30.06.2018).

Koglbauer, René \& Alina Dorota Jarzabek (im Druck): Sprachenpolitisches Handeln von Verbänden. In Brigitte Forster Vosicki, Cornelia Gick \& Thomas Studer (Hrsg.), IDT 2017. Band 3: Sprachenpolitik: Expertenberichte und Freiburger Resolution. Berlin: Erich Schmidt.

Krumm, Hans-Jürgen (2007): Sprachenpolitik. In Karl-Richard Bausch, Herbert Christ \& HansJürgen Krumm (Hrsg.), Handbuch Fremdsprachenunterricht. Tübingen: de Gruyter.

Krumm, Hans-Jürgen (2018): Vom Umgang mit der zunehmenden sprachlichen Heterogenität der Schülerinnen und Schüler: Sprachsensible Schulentwicklung und Unterrichtsgestaltung. In Marianne Hepp \& Martina Nied Curcio (Hrsg.), Educazione plurilingue. Ricerca, didattica e politiche linguistiche, 37-50. Rom: Istituto Italiano di Studi Germanici.

Österreichischer Verband für Deutsch als Fremdsprache/Zweitsprache/ÖDaF: http://www.oedaf.at. Leitlinien des ÖDaF: http://www.oedaf.at/site/wirueberuns. Strobler Thesen zur Mehrsprachigkeit des ÖDaF: www.oedaf.at/stroblerthesen (30.06.2018).

Pfeiffer, Waldemar (2015): Multilingualität und Multikulturalität. In Marina Foschi \& Konrad Ehlich (Hrsg.), Deutsch als Fremdsprache als transkultureller Erfahrungsraum. Zur Konzeptentwicklung eines Faches, 11-29. Münster: Waxmann.

Shafer, Naomi \& Martin Baumgartner (im Druck): Die Pluralität von DaF als Plus: Zu einem weitergedachten DACH-Prinzip. In Brigitte Forster Vosicki, Cornelia Gick \& Thomas Studer (Hrsg.), IDT 2017. Band 3: Sprachenpolitik: Expertenberichte und Freiburger Resolution. Berlin: Erich Schmidt.

Sorger, Brigitte (2012): Der internationale Deutschlehrerverband und seine Sprachenpolitik. Ein Beitrag zur Fachgeschichte von Deutsch als Fremdsprache. Innsbruck u.a: StudienVerlag.

Verein der Lehrenden in Deutsch als Fremd- und Zweitsprache (DaF/DaZ) an Hochschulen in der Schweiz/Ledafids: https://www.ledafids.ch/ (30.06.2018). 
Brought to you by | Universita di Pisa Authenticated Download Date | 6/28/19 12:40 PM 